\title{
Downregulation of AATK mediates microRNA-558-induced resistance of A549 cells to radiotherapy
}

\author{
RUI-XIA ZHU, CHUN-HUI SONG, JIN-SHAN YANG, QING-TING YI, BAO-JIAN LI and SI-HAI LIU \\ Department of Oncology, Central Hospital of Zaozhuang Mineral Group, Zaozhuang, Shandong 277800, P.R. China
}

Received May 19, 2015; Accepted July 4, 2016

DOI: $10.3892 / \mathrm{mmr} .2016 .5579$

\begin{abstract}
The deregulation of microRNAs (miRNAs) is often implicated in the control of sensitivity to radiotherapy. The objective of the present study was to identify the association between miR-558 and apoptosis-associated tyrosine kinase (AATK), and their importance in regulating the development of resistance to radiotherapy. The current study demonstrated that AATK, a radiosensitization-associated gene, is a target of miR-558 in lung cancer cells, using in silico analysis and a luciferase reporter system. Furthermore, it was determined that transfection of 30 or $50 \mathrm{nM}$ miR-558 mimics and AATK specific siRNA markedly suppressed the mRNA and protein expression of AATK. To determine whether miR-558 was required for lung cancer cell radioresistance, A549 cells were treated with different doses of ionizing radiation, from 0 to $10 \mathrm{~Gy}$, following transfection with miR-558 mimics or AATK specific siRNA. It was determined that the administration of miR-558 mimics or AATK specific siRNA alone did not significantly alter the survival rate of the cells. By contrast, in the cells exposed to 4, 6 or $8 \mathrm{~Gy}$, the administration of miR-558 mimics or AATK specific siRNA significantly promoted cell survival rate and overexpression of AATK reversed this effect. In conclusion, these data demonstrate that the miR-558/AATK cascade is important for the radiosensitization of lung cancer cells and may be a potential radiotherapy target.
\end{abstract}

\section{Introduction}

Greater than 1,600,000 new cases of lung cancer occur each year worldwide. Lung cancer accounts for greater than $1,370,000$ deaths annually and has become the leading cause of cancer-associated mortality. In China, lung cancer is the most common malignancy, and the incidence and mortality rates, based on ages, were higher compared with the worldwide

Correspondence to: Dr Si-Hai Liu, Department of Oncology, Central Hospital of Zaozhuang Mineral Group, 20 Qilianshan Road, Zaozhuang, Shandong 277800, P.R. China

E-mail: sihai_1@163.com

Key words: apoptosis-associated tyrosine kinase, microRNA-558, radioresistance, non-small cell lung cancer average (1). Among the various subtypes of lung cancer, non-small cell lung cancer (NSCLC) is the most common and accounts for $80 \%$ of lung cancer mortality (2). The 5 year overall survival rate for patients with NSCLC is less than $15 \%$ (3). Nearly $40 \%$ of patients with confirmed NSCLC have stage-III diseases when admitted to the hospital (4).

With the development of new technology and improved understanding of the underlying molecular mechanisms of the disease, the management of NSCLC has been greatly improved over the recent decades $(5,6)$. However, it is currently difficult to manage patients with NSCLC. Radiotherapy has been considered to be the primary treatment for NSCLC. Unfortunately, the effectiveness of radiotherapy is often limited by resistance $(2,7)$. Increasing evidence from clinical studies indicates that the response to radiotherapy varies between patients, ranging from complete recovery to relapse and distant metastasis due to resistance to radiotherapy. Additionally, normal tissues are exposed to inevitable toxic effects as a result from increasing radiation doses for treatment of radioresistant malignant cells (8-10). Resistance to radiotherapy is common in NSCLC cells (11), leading to induction of the local relapse of NSCLC $(12,13)$. Radioresistance is most likely the result of tumor heterogeneity regarding cell of origin, etiology, pathology, and genetic/molecular pathogenesis (14). Therefore, it is necessary to develop new therapeutic measures for the treatment of NSCLC, including targeted gene therapy as a radiosensitizer in order to treat the disease and increase the survival rate of patients.

MicroRNAs (miRNAs) are small endogenous single-stranded non-coding RNA molecules. They act as a negative regulator of gene expression at the post-transcriptional level by either inhibiting translation of mRNA into functional protein or accelerating the degradation of the target mRNA (15). According to Sanger miRBase, greater than 2,000 miRNAs have been identified in the human genome. They have been reported to be essential in the control of various features in cancer, including the response to chemotherapy and radiotherapy by regulating apoptosis or cell cycle (16). Apoptosis-associated tyrosine kinase 1 (AATK1), also known as lemur kinase 1, was first identified as a protein that was significantly increased during final differentiation and apoptosis of 32Dcl3, which is a non-tumorigenic mouse myeloid cell line (17). AATK is a key regulator in the control of apoptosis, which mediates the toxicity of radiotherapy towards cells (18). Previous reports determined that AATK was negatively associated with the activity of SRC proto-oncogene, 
non-receptor tyrosine kinase (Src) in lung cancer (19), and Src is key in the occurrence of radiotherapy resistance in NSCLC (20). AATK has been determined to be subject to the regulation by miRNAs (21). The present study explored potential miRNAs that regulate the expression of AATK and their importance in the control of apoptosis induced by radiation exposure.

\section{Materials and methods}

Cell culture. The A549 lung cancer cell line was obtained from the Chinese Academy of Sciences Cell Bank (Shanghai, China), and incubated in Dulbecco's modified Eagle's medium supplemented with $100 \mu \mathrm{g} / \mathrm{ml}$ streptomycin, $100 \mathrm{U} / \mathrm{ml}$ penicillin and $10 \%$ fetal bovine serum (Invitrogen; Thermo Fisher Scientific, Inc., Waltham, MA, USA) at $37^{\circ} \mathrm{C}$ in an atmosphere of $5 \% \mathrm{CO}_{2}$ in a humidified incubator. Cells the logarithmic phase of proliferation were used for the transfection.

Cell transfection. For the transfection, $1 \times 10^{6}$ cells were treated with miR-558 mimics, anti-AATK siRNA or the scramble sequence (Shanghai GenePharma Co., Ltd., Shanghai, China). The transfection was performed using $1 \mu 1$ Lipofectamine 2000 (Invitrogen; Thermo Fisher Scientific, Inc.) as specified by the manufacturer's protocol.

Reverse transcription-quantitative polymerase chain reaction $(R T-q P C R)$. The total RNA was extracted using a RNeasy Mini kit (Qiagen, Hilden, Germany). Equal amounts of total RNA (30 ng) from each treatment group (treated with either miR-558 mimics, anti-AATK siRNA or scramble) were used for cDNA synthesis using miRNA specific primers purchased from Applied Biosystems (Thermo Fisher Scientific, Inc.) according to the manufacturer's protocol. The PCR cycles were as follows: $38^{\circ} \mathrm{C}$ for $15 \mathrm{~min}, 85^{\circ} \mathrm{C}$ for $5 \mathrm{sec}$ for reverse transcription, followed by 40 cycles of $95^{\circ} \mathrm{C}$ for $10 \mathrm{sec}, 95^{\circ} \mathrm{C}$ for $5 \mathrm{sec}$ and $61^{\circ} \mathrm{C}$ for $20 \mathrm{sec}$. A TaqMan gene expression assay kit (Applied Biosystems; Thermo Fisher Scientific, Inc.) was used to determine the relative expression levels of miR-558, AATK and U6 (used as an internal control). RT-qPCR was conducted for 40 cycles in standard mode on the ABI 7900 HT Thermal cycler (Applied Biosystems; Thermo Fisher Scientific, Inc.). The reaction conditions were as follows: 40 cycles for $10 \mathrm{~min}$ at $50^{\circ} \mathrm{C}, 10 \mathrm{~min}$ at $95^{\circ} \mathrm{C}, 15 \mathrm{sec}$ at $95^{\circ} \mathrm{C}$ and $45 \mathrm{sec}$ at $60^{\circ} \mathrm{C}$. The $2^{-\Delta \Delta \mathrm{Cq}}$ method was used to calculate the relative miRNA expression values (22).

Western blot analysis. Following lysis in lysis buffer [150 mM $\mathrm{NaCl}, 20$ mM Tris-HCl pH 7.4, 1 mM EGTA, 0.1 mM EDTA, $1 \%$ Triton X-100, 2 mM NaF, Complete Protease Inhibitor Mix and $2 \mathrm{mM}$ sodium orthovanadate (Roche Diagnostics $\mathrm{GmbH}$, Mannheim, Germany)] for $20 \mathrm{~min}$ on ice, cells were centrifuged at $16,000 \mathrm{x} \mathrm{g}$ at $4^{\circ} \mathrm{C}$. A $10 \%$ SDS-PAGE gel was used to resolve proteins $(30 \mu \mathrm{g})$. Next, proteins were transferred onto nitrocellulose membranes. Subsequently, 5\% nonfat dry milk in Tris-buffered saline Tween-20 (100 mM NaCl, 0.05\% Tween-20 and $10 \mathrm{mM}$ Tris- $\mathrm{HCl} \mathrm{pH}$ 7.5) was used to block proteins to avoid non-specific binding. Following incubation with a primary anti-AATK antibody (1:5,000; cat. no. 87141; Cell Signaling Technology, Inc., Beverly, MA, USA) and anti- $\beta$-actin (1:10,000; cat. no. 3700; Cell Signaling Technology,
Inc.) for $12 \mathrm{~h}$ at $4^{\circ} \mathrm{C}$, the blots were washed and cultured with horseradish peroxidase-conjugated secondary antibodies (1:12,000; cat. no. sc-53322; Santa Cruz Biotechnology, Inc., Santa Cruz, CA, USA) for $1 \mathrm{~h}$ at $37^{\circ} \mathrm{C}$. The proteins were visualized using an ECL chemiluminescence kit (GE Healthcare Life Sciences, Chalfont, UK) according to the manufacturer's protocol and exposed to X-ray film. The signal was determined using an Odyssey scanner (LI-COR Biosciences, Lincoln, NE, USA) and signals were normalized against that of $\beta$-actin.

Ionizing radiation. At $48 \mathrm{~h}$ after the transfection, $\gamma$-ray ionizing radiation (IR) from a ${ }^{60} \mathrm{Co}$ source was used to radiate sub-confluent cell monolayers at a rate of 2, 4, 6 and $8 \mathrm{~Gy} / \mathrm{min}$.

3-(4,5-dimethylthiazol-2-yl)-2,5-diphenyltetrazolium bromide (MTT) assay. Cells $(200 \mu \mathrm{l})$ were plated on a 96-well microtiter plate at a density of $5 \times 10^{4}$ cells/ml $24 \mathrm{~h}$ prior to IR. Three days following IR, each well was supplemented with $10 \mu \mathrm{l}$ MTT reagent (Sigma-Aldrichm St. Louis, MO, USA) and incubated at $37^{\circ} \mathrm{C}$ for $4 \mathrm{~h}$. The supernatants were removed and $100 \mu \mathrm{l}$ dimethyl sulphoxide was added to terminate the reaction and the plates were mixed for $10 \mathrm{~min}$. The absorbance was determined at $490 \mathrm{~nm}$. Each experiment was repeated three times and the mean was calculated.

Plasmid construction. Human genomic DNA was used to amplify the 3'-UTR region of AATK using a TaqMan gene expression kit (Applied Biosystems), according to the manufacturer's protocol. Following amplification, it was inserted into the pmirGLO vector (Promega Corporation, Madison, WI, USA) next to the luciferase gene to establish a luciferase reporter plasmid (pmirGLO-WT-AATK-3'UTR). A QuikChange Site-Directed Mutagenesis kit (Promega Corporation) was used to perform site-directed mutagenesis of the miR-558 target site in the AATK 3'-UTR with pmirGLO-WT-AATK-3'UTR as the template, resulting to a mutant-type reporter plasmid (pmirGLO-MUT-AATK-3'UTR). In addition, the coding sequence of AATK was amplified and subcloned into pcDNA3.0 (Invitrogen; Thermo Fisher Scientific, Inc.) to make the construct (pcDNA3-AATK) for the purpose of ectopic overexpression.

Dual-luciferase reporter assay. A549 cells were seeded in 96-well plates at a density of $1 \times 10^{5}$ cells/well and transfected with $200 \mathrm{ng}$ luciferase reporter plasmid (pmirGLO-WT-AATK3'UTR or pmirGLO-MUT-AATK-3'UTR; Promega Corporation), $50 \mathrm{nM}$ mimics, scrambled microRNA or miR-558 using Lipofectamine 2000 (Invitrogen; Thermo Fisher Scientific, Inc.). Cells were collected $48 \mathrm{~h}$ following transfection, and a dual-luciferase reporter assay (Promega Corporation) was used to determine luciferase activity.

Apoptosis. Flow cytometry was used to measure apoptosis using the Apoptest-FITC Kit with annexin V (Dako, Glostrup, Denmark) as specified in the manufacturer's protocol. Flow cytometry (LSR II, BD Biosciences, San Jose, CA, USA) was used to assess the proportion of annexin V-positive cells.

Online miRNA database search. The present study searched the online miRNA databases, including TargetScan (www. targetscan.org) and miRNA database (www.mirdb.org) to 
Hsa-miR-558 and AATK

AATK 3' UTR (375-381)

Hsa-mi R-558

AATK Mutated 3' UTR (375-381)

AATK 3' UTR (755-761)

Hsa-miR-558

AATK Mutated 3' UTR (755-761)

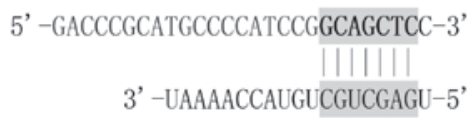

5' -GACCCGCATGCCCCATCCGCGTCGAGC-3'

Figure 1. The 3'-UTR of AATK is targeted by miR-558 with potential two 'hits' in the 3'UTR of AATK. 3'UTR, 3' untranslated region; AATK, apoptosisassociated tyrosine kinase; miR, microRNA.

predict the virtual target gene of the miRNA with the potential binding site (at least 7 consecutive complementary bases) in the 3'UTR of target mRNA.

Statistical analysis. SPSS 13.0 (SPSS, Inc., Chicago, IL, USA) was used to conduct statistical analysis. The results were presented as the mean \pm standard deviation based on three independent experiments. One way analysis of variance was used for statistical analysis. $\mathrm{P}<0.05$ was considered to indicate a statistically significant difference.

\section{Results}

$A A T K$ is a direct target of $m i R-558$. The relationship between the AATK-3'-UTR and the miRNAs targeting it was predicted through bioinformatics analysis by miRanda, which indicated that the 3'-UTR of AATK may be targeted by miR-558 with two potential 'hits' in the 3'UTR of AATK (Fig. 1). To study the interaction between AATK and miR-558, the full length of the AATK 3'UTR containing potential miR-558 binding sites or the mutants was selected for further validation using luciferase reporter assays. miR-558 mimics significantly reduced the luciferase expression in the A549 cells co-transfected with the wild type AATK 3'UTR (Fig. 2). When the binding site (375-381 bp) was mutated, the suppression of the luciferase activity was reversed, while introduction of mutations into another binding site (755-761 bp) had minimal effects (Fig. 2). These assays demonstrated that AATK is one of the target genes of miR-558, and that the 375-381 bp region, instead of the 755-761 bp region of the AATK 3'UTR is the binding site of the miRNA.

miR-558 is a regulator of AATK. To test the hypothesis that miR-558 is a direct regulator of AATK, western blotting and RT-qPCR were used to analyze differences in AATK expression in the A549 cells transfected with scrambled control and miR-558 mimics. As presented in (Fig. 3A), transfection with 30 or $50 \mathrm{nM}$ miR-558 mimics and AATK specific siRNA substantially suppressed the mRNA and protein expression levels of AATK. Transfection of the construct containing the coding sequence of AATK significantly increased the expression of AATK in A549 cells (Fig. 3B). The results further confirmed the regulatory relationship between miR-558 and AATK in lung cancer cells, and the effect of miR-558 was specific as the scrambled miRNA did not exhibit such an effect.

miR-558 is required for radioresistance of lung cancer cells. To determine whether miR-558 was required for lung cancer cell radioresistance, A549 cells were treated with different doses of ionizing radiation, from 0 to $10 \mathrm{~Gy}$, following transfection with miR-558 mimics or AATK-specific siRNA. IR treatment exhibited a dose-dependent inhibitory effect on the growth of the A549 cells (Fig. 4). In the A549 cells exposed to 2 Gy, the administration of miR-558 mimics or AATK specific siRNA did not significantly alter the survival rate of the cells (Fig. 4A). In contrast to the cells exposed to 4, 6 or $8 \mathrm{~Gy}$, administration of miR-558 mimics or AATK specific siRNA significantly promoted the survival rate of the cells, with the overexpression of AATK reversing this effect (Fig. 4B-D). This suggests that the enhanced radioresistance of the A549 cells transfected with miR-558 may be due to the downregulation of AATK. Additionally, the effect of miR-558 promotion on IR-induced apoptosis in lung cancer cells was investigated. A549 cells transfected with miR-558 mimics and AATK siRNA were exposed to IR (4 Gy), and apoptosis measured in each group. This indicated that the introduction of miR-558 or anti-AATK siRNA significantly suppressed apoptosis in lung cancer cells, while ectopic overexpression of AATK partially but significantly reversed the effect on apoptosis (Fig. 5). This suggests that the increased radioresistance may have been due to a decreased rate of apoptosis resulting from downregulation of AATK expression. In addition, the effect of exposure to radiation on the expression of AATK and miR-558 was also evaluated, with the results not indicating alterations of the miRNA and AATK expression levels (data not shown).

\section{Discussion}

It has been established that resistance to radiotherapy leads to significantly elevated mortality and morbidity of patients with NSCLC, and it is a key issue in the treatment of NSCLC. Researchers have been seeking potent approaches to sensitize cases NSCLC that are often radioresistant. 
A

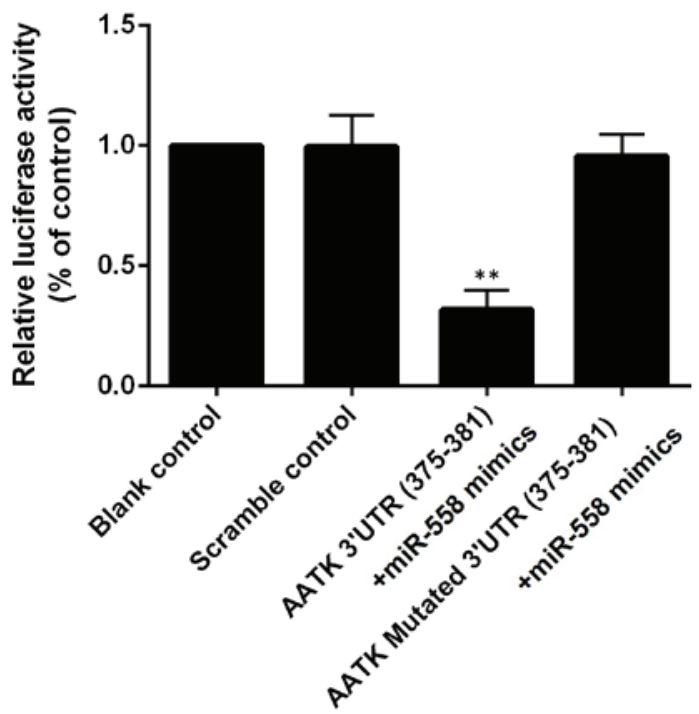

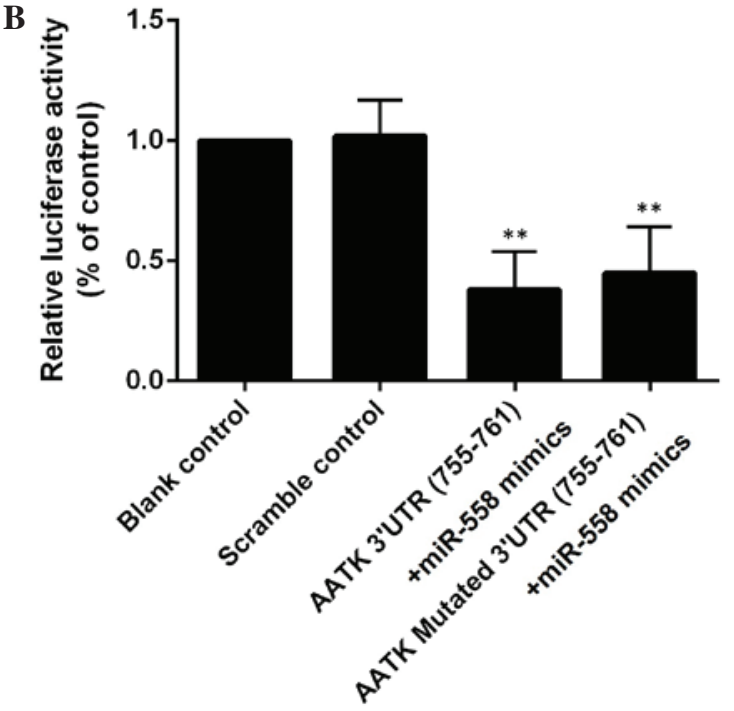

Figure 2. (A) miR-558 mimics significantly reduced luciferase expression in the cells co-transfected with the wild type AATK 3 'UTR. When the 375-38 bp binding site was mutated, the effect of miR-558 mimics was prevented. (B) Introduction of the mutations into another binding site (755-761 bp) had minimal effects. Data are presented as the mean \pm standard deviation $\left({ }^{* * *} \mathrm{P}<0.01\right)$. miR, microRNAl AATK, apoptosis-associated tyrosine kinase; $3^{\prime} \mathrm{UTR}, 3^{\prime}$ untranslated region.

$\mathbf{A}$
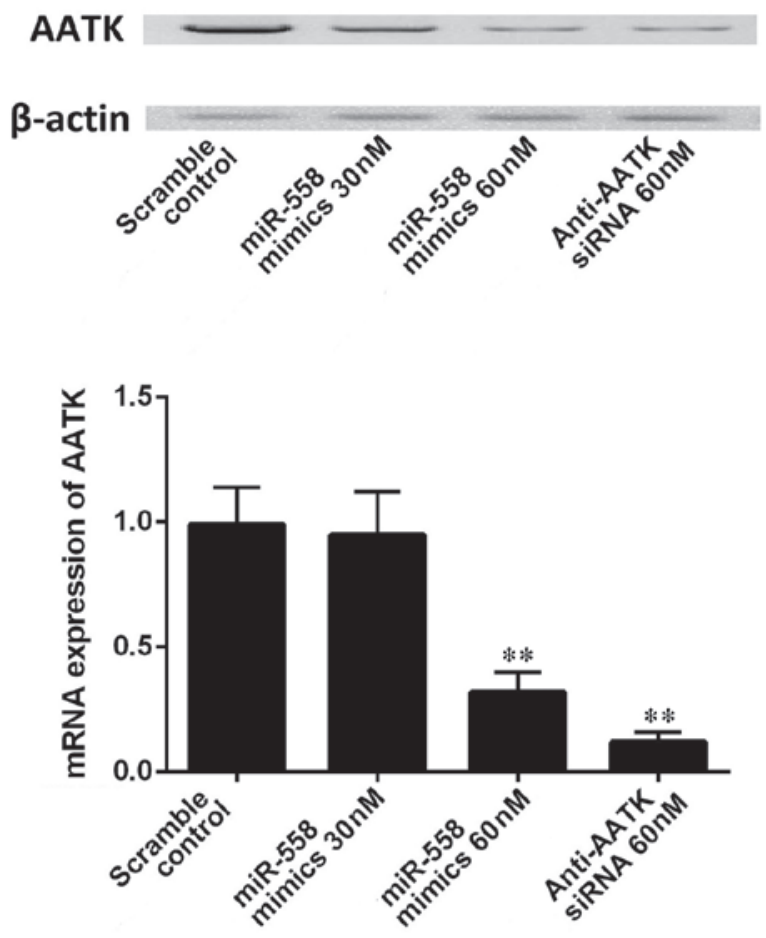

B
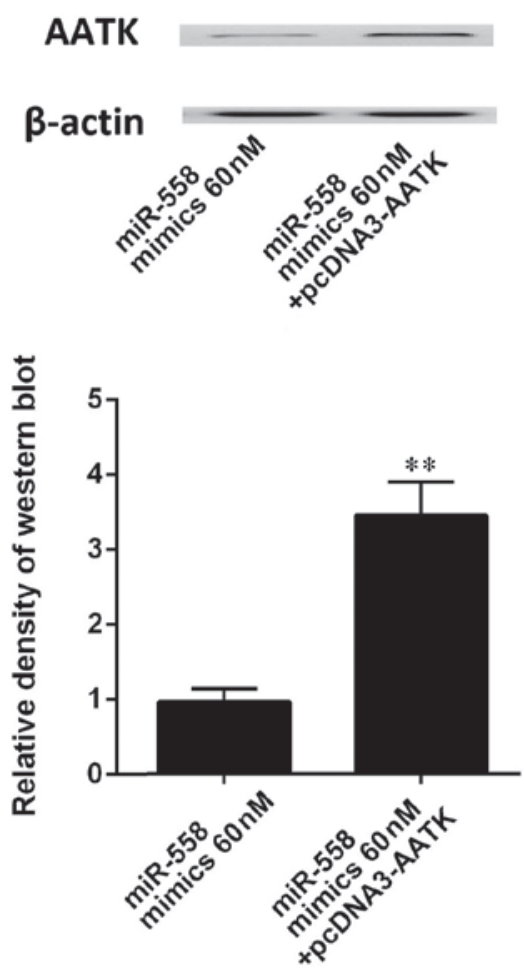

Figure 3. (A) Transfection with 30 or $50 \mathrm{nM}$ miR-558 mimics and AATK specific siRNA significantly suppressed the mRNA and protein expression of AATK. (B) Transfection with the construct containing the coding sequence of AATK significantly increased the expression of AATK in A549 cells. Data are expressed as the mean \pm standard deviation $\left({ }^{* *} \mathrm{P}<0.01\right)$. miR, microRNA; AATK, apoptosis-associated tyrosine kinase; siRNA, small interfering RNA.

AATK was identified to be significantly increased during final differentiation and apoptosis of 32Dcl3 cells, a non-tumorigenic mouse myeloid cell line (23). High expression of AATK predominantly occurs in different regions of the brain and it is detected in other tissues, including the kidney, lung, heart and skeletal muscle $(23,24)$. The focus of functional studies of AATK has been placed on the nervous system. A previous study determined that during retinoic acid-induced neuronal differentiation of P19 embryonal carcinoma cells, the expression of AATK was significantly elevated (25). Previous reports have indicated that AATK is negatively associated with the activity of Src in lung cancer (19). Src is a member of the non-receptor tyrosine kinase family, which consists of nine members, including Fyn, Lyn, Src, Fgr, Yes, Yrk, Hck, Blk and Lck. Src is functionally associated with numerous cytokine and growth factor receptors and is important in the regulation of multiple cellular activities, including cell migration, 


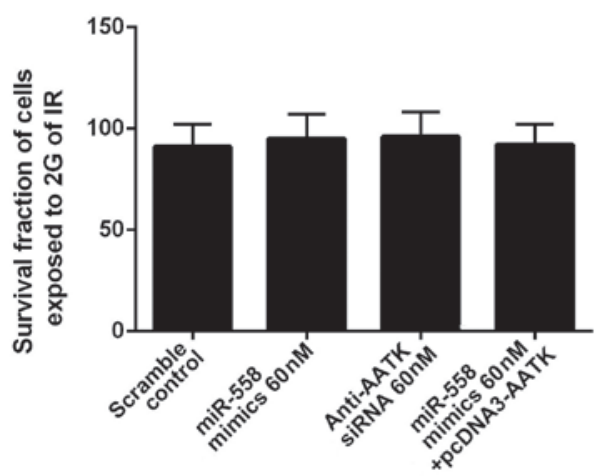

C

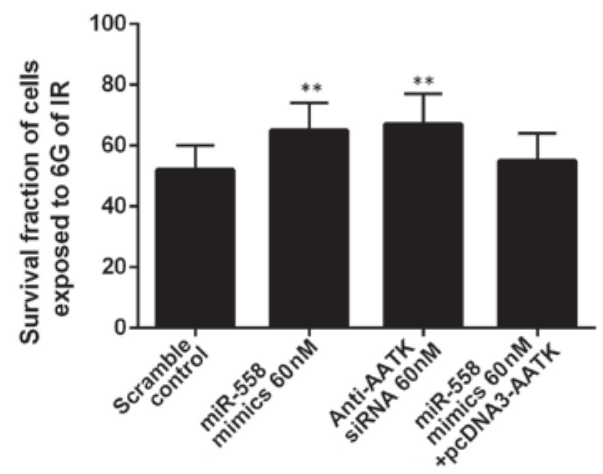

B

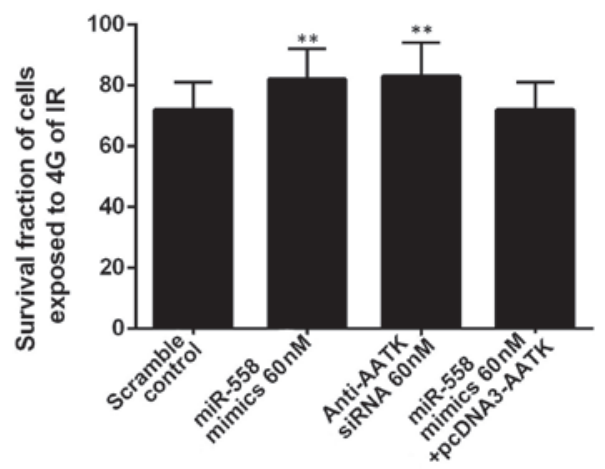

D

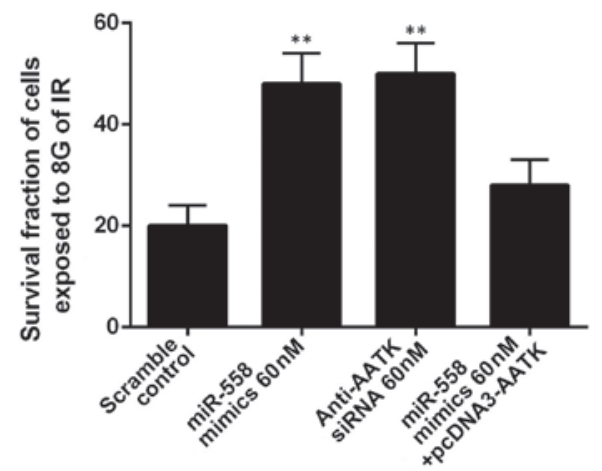

Figure 4. Ionizing radiation treatment exhibited a dose-dependent inhibitory effect on the growth of the A549 cells as determined using an MTT assay. (A) In A549 cells exposed to $2 \mathrm{~Gy}$, the administration of miR-558 mimics or AATK specific siRNA did not significantly alter the survival rate of the cells. In the cells exposed to (B) $4 \mathrm{~Gy}$, (C) 6 Gy or (D) 8 Gy, administration of miR-558 mimics or AATK specific siRNA significantly promoted the survival rate of the cells, and overexpression of AATK reduced this effect. Data are presented as the mean \pm standard deviation $\left({ }^{* *} \mathrm{P}<0.01\right.$ compared with the control). miR, microRNA; AATK, apoptosis-associated tyrosine kinase; siRNA, small interfering RNA.

A

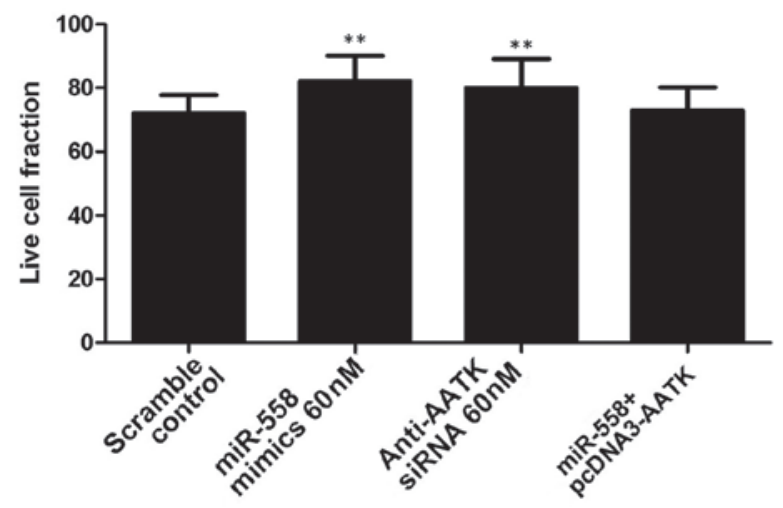

B

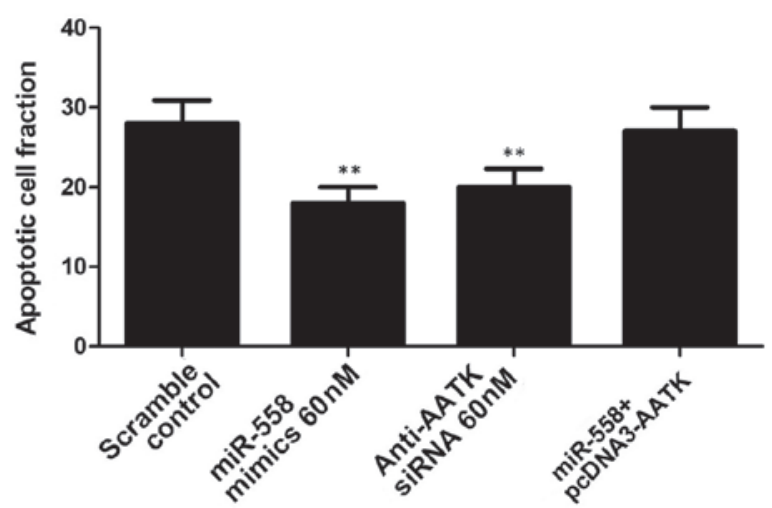

Figure 5. Introduction of miR-558 mimics or anti-AATK siRNA significantly suppressed apoptosis in lung cancer cells, while ectopic overexpression of AATK significantly reversed this effect on apoptosis. (A) Live cell fraction. (B) Apoptotic cell fraction. Data are presented as the mean \pm standard deviation (" $\mathrm{P}<0.01$ compared with the control). miR, microRNA; AATK, apoptosis-associated tyrosine kinase; siRNA, small interfering RNA.

invasion, adhesion, proliferation, survival, inflammation and angiogenesis (26). Notably, Src is commonly upregulated in lung cancer. Immunohistochemical analysis performed in a previous study indicated that the levels of Src were increased in 50-80\% of NSCLC samples tested, and Src overexpression was significantly associated with poor differentiation (27). Src is key for the occurrence of radiotherapy resistance in NSCLC (20). Activating mutations of Src have been reported in colon cancer; however, no activating mutations and gene amplifications have been identified in lung cancer $(28,29)$. Therefore, other regulators are potentially involved in the control of ATKK expression in NSCLC. The present study determined that AATK, a radiosensitization-related gene, is a target of miR-558 in lung cancer cells by using in silico analysis and luciferase reporter system. Additionally, it was identified that transfection of 30 or $50 \mathrm{nM}$ miR-558 mimics and AATK specific siRNA significantly suppressed both the mRNA and protein expression levels of AATK.

Various miRNAs have been associated with the response to radiotherapy in different tumor types, such as miR-17-92 cluster, which interferes with the response of human mantle cell lymphoma to radiotherapy (30). miR-181 sensitizes human 
gliomas to radiotherapy by acting on Bcl-2 (31). miR-221/miR-222 has been demonstrated to modulate the response of gastric carcinoma cells to radiotherapy through phosphatase and tensin homologue (32). It has been demonstrated that excessive expression of let-7 $\mathrm{g}$ and miRNA-9 in H1299 lung cancers cells leads to sensitization to radiotherapy by impeding nuclear factor- $\kappa \mathrm{B}$ pro-survival cascades (33). miR-558 is a miRNA that has been recently found in human cells. The upregulation of miR-558 has been observed in irradiated fibroblasts (34). It also has a regulatory effect on target genes associated with cell cycle checkpoints and apoptosis (35). In a previous study, significant underexpression of miR-558 was observed in breast cancer tissues (36). It has been determined that miR-558 in neuroblastoma tissues and cell lines was upregulated compared with in normal dorsal ganglia, with high expression of baculoviral IAP repeat containing 6, the host gene of miR-558, reported in a publicly available neuroblastoma microarray database (36). It has been previously determined that miR-558 acts as oncogene to increase colony formation, in vivo growth and in vitro proliferation of neuroblastoma cells (37). The current study exposed A549 cells to different doses of ionizing radiation, from 0 to $10 \mathrm{~Gy}$, following transfection with miR-558 mimics or AATK specific siRNA, and determined that the administration of miR-558 mimics or AATK specific siRNA did not significantly alter the survival rate of the cells. In contrast to the cells exposed to 4, 6 or $8 \mathrm{~Gy}$, administration of miR-558 mimics or AATK-specific siRNA significantly promoted the survival rate of the cells, and overexpression of AATK reduced this effect.

In conclusion, ATKK was validated as a target gene of miR-558, and upregulation of miR-558 was observed in radioresistant lung cancer cells. Additionally, ectopic overexpression of ATKK partially but significantly reduced the miR-558-induced radioresistance. The current study provides insight into miRNA-regulated sensitivity to radiotherapy in NSCLC and indicates that miR-558 may be a feasible novel target for the treatment of human NSCLC. Further in vivo experiments in animal models are required in the future to determine the efficacy of miR-558.

\section{References}

1. Sun X, Liu W, Wu S, Han H, Lin Y and Dai X: The morbidity and mortality trend and prediction of lung cancer in residents of Nangang District of Harbin in China during the past 10 years. Zhongguo Fei Ai Za Zhi 8, 514-517, 2005.

2. Siegel R, Naishadham D and Jemal A: Cancer statistics, 2012. CA Cancer J Clin 62: 10-29, 2012.

3. Fidias P and Novello S: Strategies for prolonged therapy in patients with advanced non-small-cell lung cancer. J Clin Oncol 28: 5116-5123, 2010 .

4. Whitehurst AW, Bodemann BO, Cardenas J, Ferguson D, Girard L, Peyton M, Minna JD, Michnoff C, Hao W, Roth MG, et al: Synthetic lethal screen identification of chemosensitizer loci in cancer cells. Nature 446: 815-819, 2007.

5. Blackstock AW and Govindan R: Definitive chemoradiation for the treatment of locally advanced non small-cell lung cancer. J Clin Oncol 25: 4146-4152, 2007.

6. Salama JK and Vokes EE: New radiotherapy and chemoradiotherapy approaches for non-small-cell lung cancer. J Clin Oncol 31: 1029-1038, 2013.

7. Le Pechoux C: Role of postoperative radiotherapy in resected non-small cell lung cancer: A reassessment based on new data. Oncologist 16: 672-681,2011.

8. Barcellos-Hoff MH, Park C and Wright EG: Radiation and the microenvironment-tumorigenesis and therapy. Nat Rev Cancer 5: $867-875,2005$
9. Madani I, De Neve W and Mareel M: Does ionizing radiation stimulate cancer invasion and metastasis? Bull Cancer 95: 292-300, 2008

10. McBride WH, Chiang CS, Olson JL, Wang CC, Hong JH, Pajonk F, Dougherty GJ, Iwamoto KS, Pervan M and Liao YP: A sense of danger from radiation. Radiat Res 162: 1-19, 2004.

11. Rödel F, Hoffmann J, Distel L, Herrmann M, Noisternig T, Papadopoulos T, Sauer R and Rödel C: Survivin as a radioresistance factor and prognostic and therapeutic target for radiotherapy in rectal cancer. Cancer Res 65: 4881-4887, 2005.

12. Lee S, Lim MJ, Kim MH, Yu CH, Yun YS, Ahn J and Song JY: An effective strategy for increasing the radiosensitivity of human lung cancer cells by blocking Nrf2-dependent antioxidant responses. Free Radic Biol Med 53: 807-816, 2012.

13. Provencio M, Sánchez A, Garrido P and Valcárcel F: New molecular targeted therapies integrated with radiation therapy in lung cancer. Clin Lung Cancer 11: 91-97, 2010.

14. Danesi R, Pasqualetti G, Giovannetti E, Crea F, Altavilla G, Del Tacca M and Rosell R: Pharmacogenomics in non-small-cell lung cancer chemotherapy. Adv Drug Deliv Rev 61: 408-417, 2009.

15. Hutvagner $\mathrm{G}$ and Zamore PD: A microRNA in a multiple-turnover RNAi enzyme complex. Science 297: 2056-2060, 2002.

16. Ketting RF, Fischer SE, Bernstein E, Sijen T, Hannon GJ and Plasterk RH: Dicer functions in RNA interference and in synthesis of small RNA involved in developmental timing in C. elegans. Genes Dev 15: 2654-2659, 2001.

17. Westermann $\mathrm{F}$ and Schwab M: Genetic parameters of neuroblastomas. Cancer Lett 184: 127-147, 2002.

18. Dewey WC, Ling CC and Meyn RE: Radiation-induced apoptosis: Relevance to radiotherapy. Int J Radiat Oncol Biol Phys 33: 781-796, 1995.

19. Ma S and Rubin BP: Apoptosis-associated tyrosine kinase 1 inhibits growth and migration and promotes apoptosis in melanoma. Lab Invest 94: 430-438, 2014.

20. Purnell PR, Mack PC, Tepper CG, Evans CP, Green TP, Gumerlock PH, Lara PN, Gandara DR, Kung HJ and Gautschi O: The Src inhibitor AZD0530 blocks invasion and may act as a radiosensitizer in lung cancer cells. J Thorac Oncol 4: 448-454, 2009.

21. Kos A, Olde Loohuis NF, Wieczorek ML, Glennon JC, Martens GJ, Kolk SM and Aschrafi A: A potential regulatory role for intronic microRNA-338-3p for its host gene encoding apoptosis-associated tyrosine kinase. PLoS One 7: e31022, 2012.

22. Livak KJ and Schmittgen TD: Analysis of relative gene expression data using real-time quantitative PCR and the 2(-Delta Delta C(T)) method. Methods 25: 402-408, 2001

23. Adie EA, Adams RR, Evans KL, Porteous DJ and Pickard BS: SUSPECTS: Enabling fast and effective prioritization of positional candidates. Bioinformatics 22: 773-774, 2006.

24. Vlodavsky I, Ilan N, Naggi A and Casu B: Heparanase: Structure, biological functions and inhibition by heparin-derived mimetics of heparan sulfate. Curr Pharm Des 13: 2057-2073, 2007.

25. Zetser A, Bashenko Y, Edovitsky E, Levy-Adam F, Vlodavsky I and Ilan N: Heparanase induces vascular endothelial growth factor expression: Correlation with p38 phosphorylation levels and Src activation. Cancer Res 66: 1455-1463, 2006.

26. Parsons SJ and Parsons JT: Src family kinases, key regulators of signal transduction. Oncogene 23: 7906-7909, 2004.

27. Mazurenko NN, Kogan EA, Zborovskaya IB and Kisseljov FL: Expression of pp60c-src in human small cell and non-small cell lung carcinomas. Eur J Cancer 28: 372-377, 1992.

28. Irby RB, Mao W, Coppola D, Kang J, Loubeau JM, Trudeau W, Karl R, Fujita DJ, Jove R and Yeatman TJ: Activating SRC mutation in a subset of advanced human colon cancers. Nat Genet 21: 187-190, 1999.

29. Kiefer PE, Bepler G, Kubasch M and Havemann K: Amplification and expression of protooncogenes in human small cell lung cancer cell lines. Cancer Res 47: 6236-6242, 1987.

30. Navarro A, Beà S, Fernández V, Prieto M, Salaverria I, Jares $P$, Hartmann E, Mozos A, López-Guillermo A, Villamor N, et al: MicroRNA expression, chromosomal alterations and immunoglobulin variable heavy chain hypermutations in mantle cell lymphomas. Cancer Res 69: 7071-7078, 2009.

31. Chen G, Zhu W, Shi D, Lv L, Zhang C, Liu P and Hu W: MicroRNA-181a sensitizes human malignant glioma U87MG cells to radiation by targeting Bcl-2. Oncol Rep 23: 997-1003, 2010.

32. Chun-Zhi Z, Lei H, An-Ling Z, Yan-Chao F, Xiao Y, Guang-Xiu W, Zhi-Fan J, Pei-Yu P, Qing-Yu Z and Chun-Sheng K: MicroRNA-221 and microRNA-222 regulate gastric carcinoma cell proliferation and radioresistance by targeting PTEN. BMC Cancer 10: 367, 2010. 
33. Arora H, Qureshi R, Jin S, Park AK and Park WY: miR-9 and let-7g enhance the sensitivity to ionizing radiation by suppression of NFkB1. Exp Mol Med 43: 298-304, 2011.

34. Cummins JM, He Y, Leary RJ, Pagliarini R, Diaz LA Jr, Sjoblom T, Barad O, Bentwich Z, Szafranska AE, Labourier E, et al: The colorectal microRNAome. Proc Natl Acad Sci USA 103: 3687-3692, 2006.

35. Maes OC, An J, Sarojini H, Wu H and Wang E: Changes in MicroRNA expression patterns in human fibroblasts after low-LET radiation. J Cell Biochem 105: 824-834, 2008.
36. Si H, Sun X, Chen Y, Cao Y, Chen S, Wang H and Hu C: Circulating microRNA-92a and microRNA-21 as novel minimally invasive biomarkers for primary breast cancer. J Cancer Res Clin Oncol 139: 223-229, 2013.

37. Shohet JM, Ghosh R, Coarfa C, Ludwig A, Benham AL, Chen Z, Patterson DM, Barbieri E, Mestdagh P, Sikorski DN, et al: A genome-wide search for promoters that respond to increased MYCN reveals both new oncogenic and tumor suppressor microRNAs associated with aggressive neuroblastoma. Cancer Res 71: 3841-3851, 2011. 